\title{
Recovery of localised structure from signals with non-sparse components
}

\author{
$\begin{array}{lll}\text { R. Broughton } & \text { I. } \text { Coope }^{2} & \text { P. Renaud } \\ & 3\end{array}$ \\ R. Tappenden ${ }^{4}$
}

(Received 24 January 2011; revised 23 July 2011)

\begin{abstract}
We consider the recovery of localised structure from signals consisting of a piecewise constant structure and sparse components. A new algorithm is presented which aims to reconstruct signals of this type from a limited set of observed data. The algorithm is broken down into two subproblems which both involve minimisation of an $l_{1}$-regularised least squares problem. Numerical results are presented which demonstrate the effectiveness and efficiency of the proposed method.
\end{abstract}

\section{Contents}

\section{Introduction}

C568

http://anziamj.austms.org.au/ojs/index.php/ANZIAMJ/article/view/3911 gives this article, (c) Austral. Mathematical Soc. 2011. Published August 3, 2011. ISSN 1446-8735. (Print two pages per sheet of paper.) Copies of this article must not be made otherwise available on the internet; instead link directly to this URL for this article. 
2 A known subspace

2.1 The projection step . . . . . . . . . . . . . C570

3 Blind signal reconstruction

C572

4 The general algorithm

C573

5 Results

C574

5.1 The unknown subspace problem . . . . . . . . . . . C574

5.2 Image reconstruction . . . . . . . . . . . . . . . C575

6 Conclusion

C577

References

C578

\section{Introduction}

Digital signal and image reconstruction is important in a wide range of areas. These include medical imaging (for example magnetic resonance imaging [11] and computed tomography [14]), astronomical imaging [13], speech and audio signal reconstruction [9], and photo-acoustic imaging [12]. In many of these applications data acquisition time is limited so the aim is to reconstruct these signals or images from a reduced data set. Typically the reconstruction problem is described by the following linear model. For a given vector of observations $\mathrm{b} \in \mathbb{R}^{\mathrm{m}}$, solve

$$
\mathrm{b}=A x+\epsilon,
$$

where $A \in \mathbb{R}^{\mathfrak{m} \times n}, \in \in \mathbb{R}^{m}$ is vector of noise (usually assumed to be Gaussian), and $x \in \mathbb{R}^{n}$ is unknown.

Without any prior knowledge about the structure of the signal to be reconstructed, we must sample at the Nyquist rate (twice the maximum frequency 
present in the signal). Essentially this means that if the signal has length $n$ then the observation vector $\mathrm{b}$ in (1) should have length $2 \mathrm{n}$ for accurate reconstruction. A new mathematical framework known as compressed sensing explains that if we know that the signal to be reconstructed is sparse (has few non-zero components) then we can take many fewer samples without affecting the reconstruction quality. So in compressed sensing we assume that $m \ll n$ (that is, equation (1) represents an underdetermined system of equations) and the signal reconstruction process is posed as

$$
\arg \min _{x}\|A x-b\|_{2}^{2}+\lambda\|x\|_{1},
$$

where the quadratic loss term enforces data fidelity and the $l_{1}$-regularisation term encourages a sparse solution. Here $\lambda$ is a user defined regularisation parameter. Early work on compressed sensing $[3,6,5,4]$ shows that we only need

$$
m \geqslant c \cdot k \cdot \ln n
$$

observations of the signal to ensure accurate reconstruction, where $k$ is the number of non-zero components in $x, \boldsymbol{n}$ is the length of $x$, and $\mathrm{c}$ is a problem dependent constant.

Outline The aim of this work is to investigate whether it is possible, from a limited set of observations, to reconstruct signals which are a combination of a piecewise constant signal and sparse components. Such signals have a specific structure which we aim to exploit. Section 2 considers the reconstruction problem assuming that the piecewise constant structure of the signal is known. Then Section 3 considers the problem of signal reconstruction when the piecewise structure is unknown and must first be recovered. This 'blind' reconstruction case is considerably more difficult. Finally, Section 5 gives numerical examples which show that the reconstruction of signals with the given structure is possible to a high level of accuracy even from a limited set of data. 


\section{A known subspace}

Mathematically we wish to determine a solution to (1) of the form

$$
x=x_{s}+v,
$$

where $v$ is a piecewise constant vector and $x_{s}$ is sparse. Because $v$ is a piecewise constant vector it is decomposed as

$$
v=\sum_{i=1}^{p} \alpha_{i} u_{i} \quad(p \ll n),
$$

where the vectors $\mathfrak{u}_{1}, \ldots, \mathfrak{u}_{\mathrm{p}}$ represent the various flat box-car areas of the signal, and the coefficient $\alpha_{i}$ represents the height of the ith flat region. The vectors $\mathfrak{u}_{1}, \ldots, \mathfrak{u}_{\mathrm{p}}$ span a subspace of $\mathbb{R}^{\mathfrak{n}}$ which we denote by $M=$ $\operatorname{span}\left\{\mathfrak{u}_{1}, \ldots, \mathfrak{u}_{\mathbf{p}}\right\}$. Because we have an underdetermined system of equations, following the procedure recommended for reconstructing sparse solutions [4], the reconstruction problem is recast as

$$
\arg \min _{x_{s}, v}\left\|A x_{s}+A v-b\right\|_{2}^{2}+\lambda\left\|x_{s}\right\|_{1} .
$$

This is an unconstrained convex optimisation problem in $2 \mathrm{n}$ unknowns. To accurately reconstruct $x$, we first determine the components $v$ and $x_{s}$. Problem (4) is split into two subproblems in the following way:

$$
\arg \min _{x_{s}}\left[\left\{\arg \min _{v}\left\|A x_{s}+A v-b\right\|_{2}^{2}\right\}+\lambda\left\|x_{s}\right\|_{1}\right] .
$$

This means that to solve problems of the form (4), we instead solve each of the two subproblems described by (5) in turn.

\subsection{The projection step}

The aim now is to determine a way of removing $v$ from problem (5) so that we are solving a simpler optimisation problem. Suppose we know the 
subspace $M$, and therefore the vectors $\mathfrak{u}_{1}, \ldots, \mathfrak{u}_{p}$. This does not mean that we know the vector $v$ because we do not know $\alpha_{1}, \ldots, \alpha_{p}$ and these are not simple to determine. Define $C$ to be the matrix whose columns are the vectors $\left\{A u_{1}, A u_{2}, \ldots, A u_{p}\right\}$, and write $C=Q R$ for its economy QR decomposition [8, p.223]. The vectors $A u_{1}, \ldots, A u_{p}$ must be linearly independent for $R$ to be invertible. The columns of the matrix $Q$ represent an orthonormal basis spanning the subspace $M$, with $Q^{\top} Q=I$ on $M$. Define the matrix

$$
\mathrm{P}=\mathrm{I}-\mathrm{QQ}^{\top}
$$

which is the projection onto the orthogonal complement $M^{\perp}$. Premultiplying expression (1) by $\mathrm{P}$ gives

$$
\mathrm{Pb}=\mathrm{PA} x_{s}+\mathrm{PA} v+\mathrm{P} \epsilon=\mathrm{PA} x_{s}+\epsilon^{\prime}
$$

because PA $v=\mathbf{0}$ (the zero vector). Using the projection matrix (6) we have essentially 'removed' $A v$ from problem (4) so we now have the optimisation problem

$$
\arg \min _{\chi_{s}}\left\|P A x_{s}-P b\right\|_{2}^{2}+\lambda\left\|x_{s}\right\|_{1}
$$

Once problem (7) is solved for $x_{s}$, we recover $v$ as follows. Let $\alpha$ denote the vector whose components are $\alpha_{1}, \ldots, \alpha_{p}$. Then

$$
\mathrm{b}^{\prime}=\mathrm{b}-\mathrm{Ax} \mathrm{x}_{\mathrm{s}} \approx \mathrm{A} v=\mathrm{C} \alpha
$$

In the noiseless case, the approximation becomes equality. This is now an over determined system and so is solved using the normal equations or, because the QR decomposition has already been determined, by performing back-substitution on

$$
\mathrm{R} \alpha=\mathrm{Q}^{\top} \mathrm{b}^{\prime}
$$

Once $\alpha$ has been determined, $v$ is recovered through $v=\sum_{i=1}^{p} \alpha_{i} u_{i}$ and then the signal $x=x_{s}+v$ is known. 
Remark The matrix-vector product $\mathrm{Pb}$ is computed once before the algorithm is initialised. The multiplication $P A x_{s}$ is computed as two matrix-vector products - this means there is only one extra matrix-vector product per iteration compared with a standard compressed sensing problem formulation, described by (4).

\section{Blind signal reconstruction}

The problem of signal reconstruction when the subspace $M$ is unknown is sometimes referred to as 'blind' reconstruction and is the topic of much current research. So far we have assumed that in the decomposition $x=x_{s}+v$, the component $v$ was in a known subspace. So, in the previous section, $v$ was a piecewise constant function where the possible discontinuities were in certain known positions.

This section considers the more difficult problem of finding $v$ when the subspace $M$ is unknown, and depends on some parameters which must be found. Once found, the problem is then solved by the procedure in Section 2. A typical problem of this nature occurs when $v$ is a piecewise constant vector with jumps at unknown positions.

Because $v$ is piecewise constant, adjacent signal entries are usually the same. That is, often $v_{i}-v_{i+1}=0$. Furthermore, if $v_{i}-v_{i+1} \neq 0$, then a discontinuity has been located. Left-multiplying $v$ by the discrete derivative matrix [10]

$$
\mathrm{D}=\left[\begin{array}{ccccc}
1 & -1 & & & \\
& 1 & -1 & & \\
& & \ddots & \ddots & \\
& & & 1 & -1 \\
& & & & 1
\end{array}\right]
$$

gives the vector $v_{s}$ with entries $v_{i}-v_{i+1}$. So now

$$
v_{\mathrm{s}}=\mathrm{D} v \Rightarrow v=\mathrm{D}^{-1} v_{\mathrm{s}},
$$


If there are only a small number of discontinuities, then the vector $v_{\mathrm{s}}$ is sparse and its non-zero entries correspond to the positions of the discontinuities in $v$. Note that $v_{s} \neq x_{s}$. Since $x_{s}$ is sparse, $\left\|A x_{s}\right\| \ll\|A v\|$ which suggests the approximation

$$
\mathrm{b} \approx \mathrm{A} v=\mathrm{AD}^{-1} v_{\mathrm{s}} .
$$

Furthermore, because $v_{s}$ is sparse, it is determined using the standard compressed sensing setup

$$
\min _{v_{s}}\left\|A D^{-1} v_{s}-b\right\|_{2}^{2}+\lambda\left\|v_{s}\right\|_{1} .
$$

Once $v_{s}$ has been found, the subspace $M$ is known. However, as (9) is only an approximation, we do not accurately know the values $\alpha_{1}, \ldots, \alpha_{p}$ and, in turn, we do not accurately know $v$. So we return to the problem described in Section 2 and project out of the subspace $M$ using the projection matrix (6) and solve a second optimisation problem (7) to determine $x_{s}$. Next the values $\alpha_{1}, \ldots, \alpha_{p}$ are found, and $x$ is reconstructed.

\section{The general algorithm}

To reconstruct a signal which is a combination of a piecewise constant signal and a sparse component from a limited set of observed data requires two stages. The first stage requires a sparse vector to be found where the spikes in the signal correspond to the locations of the discontinuities in the piecewise constant signal. The second stage uses this information to construct a projection matrix which is applied to the original data so that the underlying sparse signal is found using standard compressed sensing algorithms.

The proposed algorithmic process involves the steps of Algorithm 1.

There are a considerable number of recently proposed algorithms which solve problems of the form (2). One such algorithm is the BCGP algorithm [2] which we use in all numerical results presented in the following section as it has been shown to be efficient and accurate. 
Algorithm 1: Signal reconstruction process.

1 Solve the optimisation problem $\arg \min _{v_{s}}\left\|A D^{-1} v_{s}-b\right\|_{2}^{2}+\lambda\left\|v_{s}\right\|_{1}$ which gives the parameters to determine the subspace $M$;

2 Form the matrix $\mathrm{C}$ and perform an economy QR decomposition;

3 Form the projection matrix $\mathrm{P}=\mathrm{I}-\mathrm{QQ}^{\top}$;

4 Solve the transformed problem $\arg \min _{x_{s}}\left\|P A x_{s}-P b\right\|_{2}^{2}+\lambda\left\|x_{s}\right\|_{1}$;

5 Determine the values $\alpha_{1}, \ldots, \alpha_{p}$ using $R \alpha=Q^{\top} b^{\prime}$;

6 Recover $v=\sum_{i=1}^{p} \alpha_{i} u_{i}$, and consequently $x=x_{s}+v$;

\section{$5 \quad$ Results}

This section presents numerical examples which demonstrate the practical performance of the algorithm. The algorithm was implemented in Matlab and the experiments were performed under Linux on an Intel Xeon $3.2 \mathrm{GHz}$ processor with $4 \mathrm{~GB}$ of memory.

\subsection{The unknown subspace problem}

This example considers a signal which is the sum of an underlying piecewise constant signal with unknown discontinuities, and a sparse signal. A sensing matrix $A$ of size $2000 \times 10000$ was constructed. The observation vector $\mathrm{b} \in \mathbb{R}^{2000}$ was measured in the presence of normally distributed Gaussian noise. The sparse signal had 100 spikes while the piecewise constant signal had ten 'jump' points and the height of each flat region was randomly generated.

The location and heights of the jumps in the piecewise constant function have been accurately located, even for very small jumps. This is an excellent reconstruction. The mean square error $(\mathrm{MSE})^{1}$ for the reconstructed signal is $1.8148 \times 10^{-5}$ which is small and confirms the accuracy of the reconstruction.

${ }^{1}$ We follow Figueiredo et al. [7] and define the MSE to be MSE $=\frac{1}{n}\|x-\hat{x}\|_{2}^{2}$ where $x$ is the original signal and $\hat{x}$ is the reconstructed signal. 
(a)

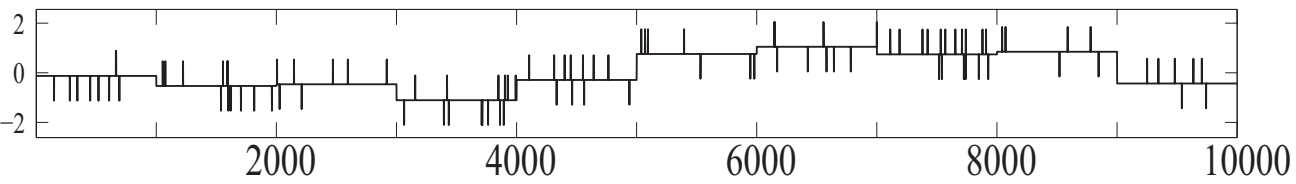

(b)

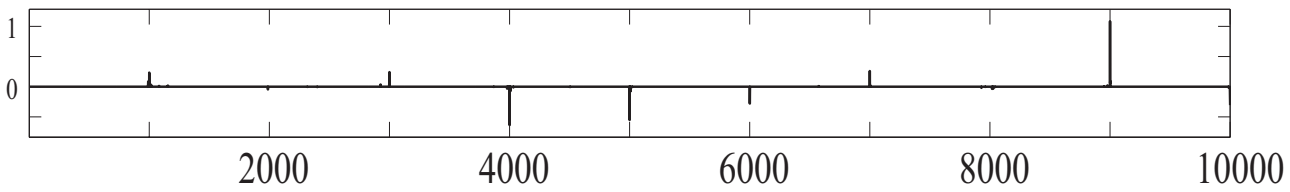

(c)

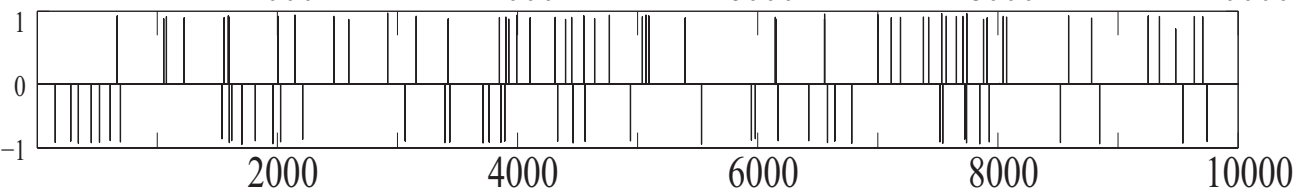

(d)

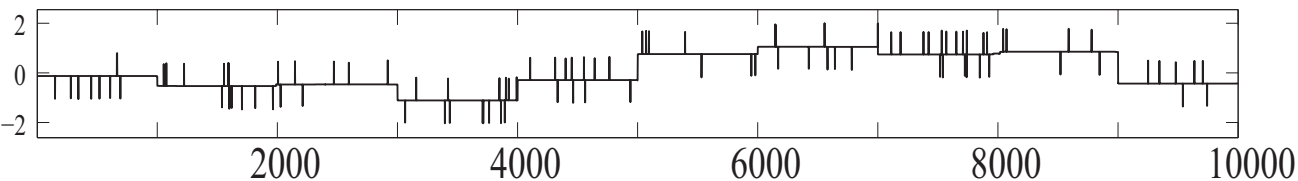

Figure 1: Signal reconstruction when the subspace $M$ is unknown: (a) the original signal; (b) the sparse signal $v_{s}$; (c) the sparse signal $x_{s}$; (d) the overall reconstruction.

\subsection{Image reconstruction}

This example considers the reconstruction of the Shepp-Logan phantom, which is a standard Matlab phantom. The phantom size is $50 \times 50$ pixels. The columns of the image are concatenated to represent the image as a column vector $x$ of length 2500 . The vector has 1204 non-zero elements so this is not a sparse image. However, Dx (where D is the matrix defined in (8)) is sparse with only 189 non-zero elements.

Recall equation (3) where $k=189$. Then $A$ is the sensing matrix with $\mathrm{m}=1479$ orthogonal rows of length 2500 and an observation vector $\mathrm{b}$ of length $\mathrm{m}$ was measured in the presence of noise. 
(a) Shepp-Logan Phantom Signal

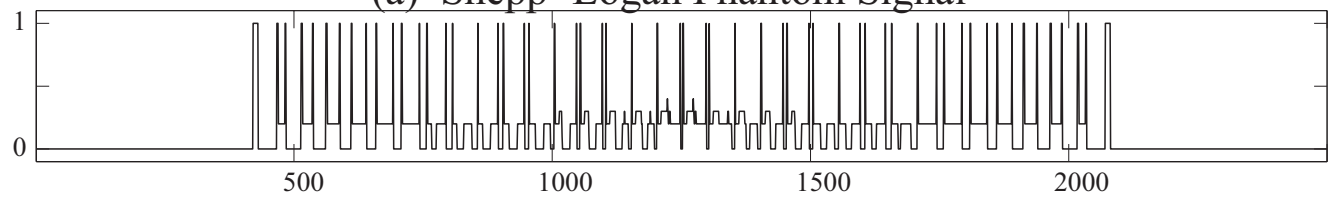

(b) Location of Jumps

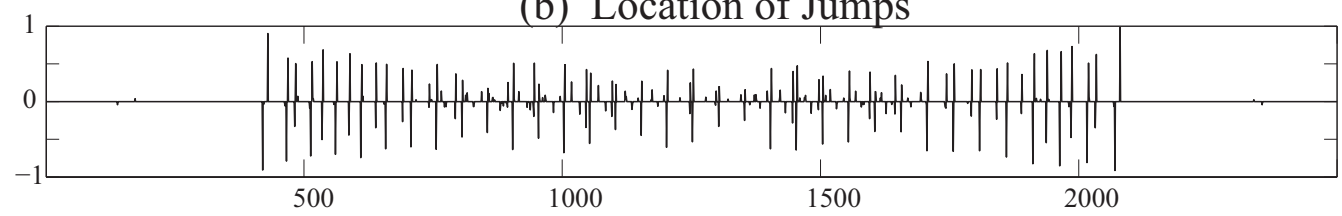

(c) Piecewise Constant Signal
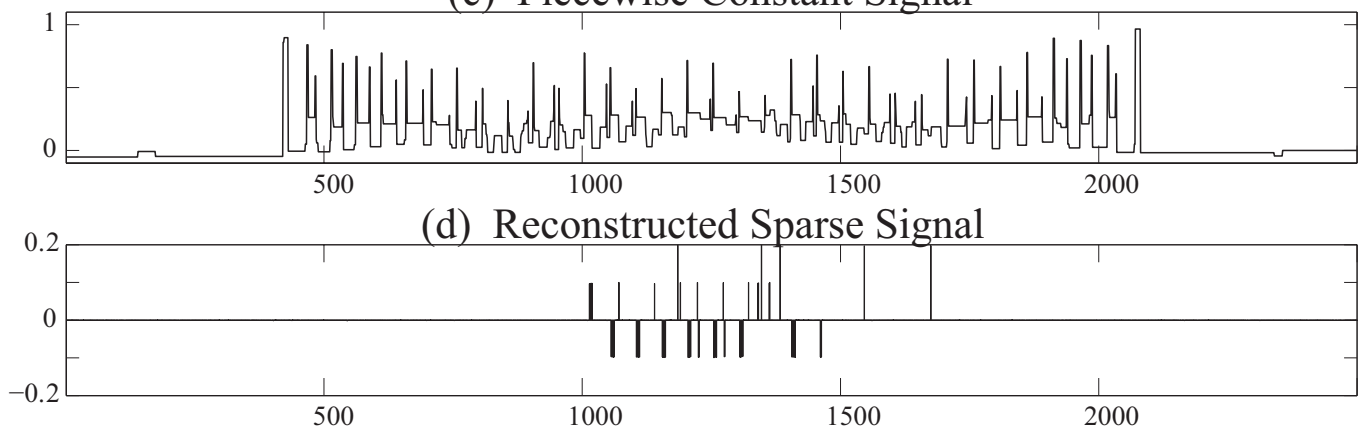

(d) Reconstructed Sparse Signal

(e) Overall Reconstruction

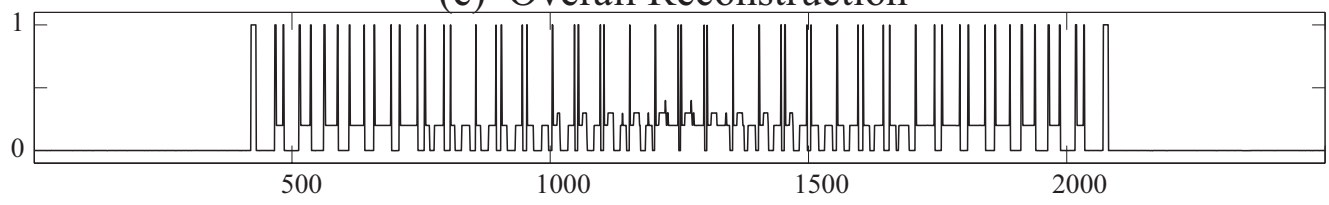

Figure 2: The reconstruction of the Shepp-Logan Phantom: (a) the original signal; (b) the sparse signal $v_{s}$; (c) the piecewise constant signal; (d) the sparse signal $\chi_{s} ;(e)$ the overall reconstruction. 
Figure 2 shows the signal evolution at various stages of the reconstruction process. Plot 2(b) shows the location of discontinuities in the piecewise constant signal (the vector $v_{\mathrm{s}}$ from equation (10)). The piecewise constant signal approximation is shown in 2(c). The reconstructed sparse signal is shown in 2(d) and shows the finer detail of the signal. The final plot, 2(e), is the overall signal reconstruction. This is an excellent reconstruction with all fine detail present. The MSE for this reconstruction is $1.37 \times 10^{-8}$ which confirms the accuracy of the final signal.

Remark Solving problem (10) takes up the bulk of total computation time with four matrix-vector products per iteration required. It is extremely fast to compute the matrix $\mathrm{C}$ and its QR decomposition. Solving the optimisation problem (7) is faster than the first stage because the algorithm typically terminates in fewer iterations, although there are still four matrix-vector products per iteration required. Nonetheless, each of the examples in this section was solved in under two minutes. Future research will focus on speeding up the first part of the reconstruction process - for example, using the cSALSA [1] algorithm which has been shown to perform very quickly.

\section{Conclusion}

The problem of reconstructing signals and images which are a combination of a piecewise constant plus sparse components is important, particularly in medical imaging. We showed that it is possible to reconstruct these types of objects from under sampled data. The reconstruction was posed as a three step process. The first and third steps involve solving an $l_{1}$-regularised least squares optimisation problem, while the second step involves finding and applying a projection matrix. We introduced an algorithm which allows signal reconstruction with no prior knowledge about the support of the signal. The numerical results show that this method gives accurately reconstructed images in a short period of time. 
Acknowledgements Rachael Tappenden is the recipient of a doctoral scholarship from the New Zealand Institute of Mathematics and its Applications. We thank the anonymous referees for providing us with constructive comments and suggestions.

\section{References}

[1] M. V. Afonso, J. M. Bioucas-Dias, and M. A. T. Figueiredo. A Fast Algorithm for the Constrained Formulation of Compressive Image Reconstruction and Other Linear Inverse Problems. IEEE International Conference on Acoustics Speech and Signal Processing, pages 4034-4037, 2010. doi:10.1109/ICASSP.2010.5495758 C577

[2] R. L. Broughton, I. D. Coope, P. F. Renaud, and R. E. H. Tappenden. A Box Constrained Gradient Projection Algorithm for Compressed Sensing. Signal Processing, 91(8):1985-1992, August 2011. doi:10.1016/j.sigpro.2011.03.003 C573

[3] E. Candès, J. Romberg, and T. Tao. Robust Uncertainty Principles: Exact Signal Reconstruction from Highly Incomplete Frequency Information. IEEE Transactions on Information Theory, 52(2):489-509, 2004. doi:10.1109/TIT.2005.862083 C569

[4] E. J. Candès. Compressive Sampling. In Proceedings of the International Congress of Mathematics, pages 1433-1452, Madrid, Spain, 2006. European Mathematical Society. C569, C570

[5] E. J. Candès and T. Tao. Near-Optimal Signal Recovery From Random Projections and Universal Encoding Strategies. IEEE Transactions on Information Theory, 52(12):5406-5425, December 2006. doi:10.1109/TIT.2006.885507 C569

[6] D. L. Donoho. Compressed Sensing. IEEE Transactions on Information Theory, 52(4):1289-1306, 2006. doi:10.1109/TIT.2006.871582 C569 
[7] M. A. T. Figueiredo, R. D. Nowak, and S. J. Wright. Gradient Projection for Sparse Reconstruction: Application to Compressed Sensing and Other Inverse Problems. IEEE Journal of Selected Topics in Signal Processing, 1(4):586-597, December 2007. doi:10.1109/JSTSP.2007.910281 C574

[8] G. H. Golub and C. F. Van Loan. Matrix Computations. The Johns Hopkins University Press, 3 edition, 1996. C571

[9] A. Griffin and P. Tsakalides. Compressed sensing of audio signals using multiple sensors. Lausanne, Switzerland, 25-29 August 2008. EUSIPCO08. C568

[10] P. C. Hansen. Rank-Deficient and Discrete Ill-Posed Problems. SIAM, 1998. C572

[11] M. Lustig, D. Donoho, and J. Pauly. Sparse MRI: The Application of Compressed Sensing for Rapid MR Imaging. Magnetic Resonance in Imaging, 58(6):1182-1195, December 2007. doi:10.1002/mrm.21391 C568

[12] J. Provost and F. Lesage. The Application fo Compressed Sensing for Photo-Acoustic Tomography. IEEE Transactions on Medical Imaging, 28(4), April 2009. doi:10.1109/TMI.2008.2007825 C568

[13] J. L. Starck and J. Bobin. Astronomical Data Analysis and Sparsity: From Wavelets to Compressed Sensing. Proceedings of the IEEE, 98(6):1021-1030, 2010. doi:10.1109/JPROC.2009.2025663 C568

[14] H. Yu and G. Wang. Compressed sensing based interior tomography. Physics in Medicine and Biology, pages 2791-2805, 2009. doi:10.1088/0031-9155/54/9/014 C568 


\section{Author addresses}

1. R. Broughton, Department of Mathematics and Statistics, University of Canterbury, Christchurch 8041, New Zealand. mailto:r.broughton@math. canterbury . ac.nz

2. I. Coope, Department of Mathematics and Statistics, University of Canterbury, Christchurch 8041, New Zealand. mailto: i.coope@math. canterbury.ac.nz

3. P. Renaud, Department of Mathematics and Statistics, University of Canterbury, Christchurch 8041, New Zealand. mailto: p.renaud@math. canterbury . ac.nz

4. R. Tappenden, Department of Mathematics and Statistics, University of Canterbury, Christchurch 8041, New ZeALAND. mailto:tappenden@math. canterbury.ac.nz 